\title{
Entre logiques de redistribution et volonté d'entreprendre : les relations complexes des migrantes sénégalaises avec leurs familles d'origine
}

The Rationale of Redistribution and the Will to Enterprise: The Complex

Relationships of Female Senegalese Migrants with their Families of Origin

Entre lógicas de redistribución y voluntad de emprender: las difíciles relaciones

de migrantes senegalesas con sus familias de origen

\section{Melissa Blanchard}

\section{OpenEdition}

\section{Journals}

Édition électronique

URL : https://journals.openedition.org/remi/5543

DOI : 10.4000/remi.5543

ISSN : $1777-5418$

Éditeur

Université de Poitiers

Édition imprimée

Date de publication : 1 octobre 2011

Pagination : 139-159

ISBN : 979-10-90426-01-6

ISSN : 0765-0752

Référence électronique

Melissa Blanchard, «Entre logiques de redistribution et volonté d'entreprendre : les relations complexes des migrantes sénégalaises avec leurs familles d'origine », Revue européenne des migrations internationales [En ligne], vol. 27 - n² | 2011, mis en ligne le 01 octobre 2014, consulté le 16 avril 2022. URL : http://journals.openedition.org/remi/5543 ; DOI : https://doi.org/10.4000/remi.5543 


\title{
Note de recherche
}

\section{Entre logiques de redistribution et volonté d'entreprendre : les relations complexes des migrantes sénégalaises avec leurs familles d'origine}

\author{
Melissa BLANCHARD ${ }^{1}$
}

$\mathrm{D}$ ans le milieu académique et au sein des institutions internationales, on souligne souvent l'importance des transferts de fonds des migrants africains vers leur pays d'origine. On relève l'ampleur de l'influence de ces rapatriements de fonds sur le développement économique local, national et régional, ainsi que sur la survie et l'amélioration des conditions des ménages (La Brosse et Mbaye, 1994 ; Daum, 1998 ; Daum, Diarra, Gonin et Philippe, 1988 ; Gonin, 2001 ; Gonin, 2005 ; Gonin et Lacroix, 2005 ; AFDB, 2007 ; Diop, 2008 ; Schmidz, 2008)².

Si nous nous situons du côté des acteurs, le discours des migrants semble calquer sur celui des rapports officiels : ils soulignent l'importance des envois d'argent et ils en expliquent la nécessité, non seulement au niveau économique, comme palliatif à la pauvreté endémique de leur pays et aux conditions de vie difficiles de leurs familles d'origine, mais aussi et surtout au niveau social. Soutenir sa famille, rembourser la dette que l'individu a

1 Chercheuse post-doc, Università degli Studi di Trento, Facoltà di Sociologia, Centro Scenari Migratori e Mutamento Sociale (SMMS), via Verdi 26, 38122 Trento, Italia ; melissa.blanchard@ unitn.it

2 Les transferts d'argent peuvent représenter une grande partie du produit intérieur brut des pays d'émigration. Le Sénégal, par exemple, se situe à la quatrième place parmi les pays africains au sud du Sahara par l'importance du montant des remises, même si les chiffres donnés sont souvent en désaccord. Par ailleurs, la véritable ampleur de ces transferts ne peut pas être restituée par les statistiques officielles disponibles, car une grande partie d'entre eux se réalise hors des circuits du système financier, empruntant plutôt des voies informelles. La Banque Mondiale estimait le montant des transferts reçus des migrants pour l'année 2001 à 174 millions de dollars (Global Development Finance, WB, 2003). Le Bureau International du Travail évaluait que ces remises correspondaient à $7 \%$ du PIB et à $82 \%$ de l'aide publique au développement du Sénégal, en 2002 . L'African Developpement Bank calcule qu'en 2005 le Sénégal a reçu 1254 millions d'euros et l'Organisation Internationale pour les Migrations (OIM) signale que 410 milliards de FCFA ont été transférés par les expatriés sénégalais dans leur pays d'origine au cours de l'année 2006. 
contractée envers le groupe social qui l'a élevé (Marie, 1997a) est une obligation morale pour les immigrés, un devoir aussi inévitable que constitutif de leur identité sociale.

Mais si la rhétorique de circonstance prône la nécessité de pourvoir aux besoins de la famille d'origine ${ }^{3}$ et vante le prestige qui en découle, la question du soutien matériel que le migrant doit à son groupe d'origine apparaît comme controversée quand on interroge les discours « de façade ». Ainsi, tout en reconnaissant l'importance de respecter les obligations qui les lient à leurs milieux d'origine, les migrants sénégalais affirment leur volonté de se libérer du devoir d'assistance envers la famille, non mesuré et non mesurable, du rôle de «patron » dans des rapports de dépendance personnelle qui s'expriment par l'envoi d'argent au pays et qui s'apparente souvent pour eux à une ponction constante et régulière.

Dans cette note de recherche, je me propose d'illustrer la complexité des rapports sociaux et matériels qui lient les sénégalaises immigrées à Marseille à leurs familles d'origine. Je partirai de l'étude des transferts d'argent et des tentatives des migrantes de constituer des partenariats entrepreneuriaux avec les membres de leur famille restés au pays, pour analyser ensuite les stratégies développées pour répondre aux demandes pressantes d'aide qui leur sont adressées, tout en essayant de préserver un espace d'autonomie, décisionnel et financier, pour elles-mêmes et pour les familles qu'elles ont fondées en France.

En m'intéressant à ces dynamiques, je serai amenée à m'interroger sur la question de la prise d'autonomie des migrantes par rapport à leurs familles et à leurs cadres sociaux d'origine. Il s'agit d'une question fondamentale qui se trouve au cœur des dynamiques actuelles de l'appréhension sociale des migrations dans les sociétés de départ (Quiminal, 1991 ; Fall, 1993 ; Quiminal, 1998 ; Streiff-Fenart, 2006). Elle peut être formulée aussi en termes d'individualisation, si l'on entend par là une prise de distance de la personne, autant sur le plan matériel que psychologique, par rapport à ses inscriptions sociales d'origine (Marie, 1997b). L'individualisation renvoie à un processus plus complexe dans le cas des femmes migrantes. L'identité de genre, en effet, détermine la trajectoire biographique des individus et a fortiori celle des migrants : elle définit leur liberté de mouvement et de décision, la restreignant parfois sensiblement, et elle leur attribue une série de rôles et de statuts qu'ils doivent endosser s'ils ne veulent pas être rejetés de la communauté migrante.

Donner la parole aux femmes migrantes et analyser leur parcours biographique me permettra d'étudier les rapports complexes qu'elles entretiennent avec les contraintes liées à leur rôle sexué, ainsi que les négociations et utilisations stratégiques auxquelles elles les soumettent, en particulier dans la gestion des rapports avec la famille d'origine. Ainsi, nous verrons que la condition de dépendance structurelle des femmes africaines à

3 Les migrants entendent généralement par là leurs parents et la fratrie, mais aussi, selon les cas, d'autres collatéraux plus éloignés. 
laquelle font référence plusieurs études ${ }^{4}$ peut être lue selon différentes perspectives : les mêmes migrantes peuvent apparaître, tour à tour, comme les figures de la dépendance ou les actrices de l'autonomie.

Plus spécifiquement, j'essaierai de déterminer quel est le lien entre l'individualisation, l'action économique entrepreneuriale des migrantes sénégalaises et leurs obligations envers la communauté d'origine. Cela m'amènera à m'interroger sur le sens de la « réussite », à savoir, de l'aboutissement de leur parcours migratoire et professionnel. Comment atteint-on la réussite en migration? Et quel est son sens ? À qui bénéficie-t-elle, à l'individu ou au groupe ? Il y a des définitions de réussite différentes qui apparaissent implicitement dans les travaux sur les entrepreneurs africains. Car si d'un côté on décrit la réussite comme la capacité d'asseoir son pouvoir social, par le biais de dépenses ostentatoires et de redistributions communautaires (Cordonnier, 1987 ; Grégoire et Labazée, 1993b ; Clark, 1994 ; Bouly de Lesdain, 1999 ; Lecarme-Frassy, 2000), de l'autre la réussite de l'entrepreneur apparaît comme l'expression de la construction d'un parcours professionnel individuel qui s'affranchit des contraintes communautaires (Lambert, 1993 ; Sengel, 2000).

\section{LES MIGRANTES SÉNÉGALAISES ENTREPRENEURES À MARSEILLE, OU L'OMNIPRÉSENCE DU COMMERCE}

Afin d'analyser les relations entre les migrantes et leurs familles d'origine, j'exposerai des études de cas issues d'une recherche que j'ai menée entre 2004 et 2007 à Marseille dans le cadre de ma thèse de doctorat. Le but de cette recherche était de comprendre si, et dans quelle mesure, le parcours migratoire et l'investissement dans le travail indépendant, notamment de type commercial, pouvaient représenter pour ces femmes des vecteurs de prise d'autonomie par rapport à leur communauté d'origine et à leur famille, aussi bien au Sénégal qu'en France.

Avant toute autre considération, il est nécessaire de remarquer que suite au recensement des principales activités entrepreneuriales des migrantes sénégalaises à Marseille, le commerce a rapidement émergé comme l'activité indépendante dominante. Les commerçantes « stables » (installées autant sur les marchés qu'en boutique et dans des espaces de vente informels) qui exercent à Marseille sont une cinquantaine, auxquelles il faut ajouter un nombre sensiblement plus élevé de femmes qui font du commerce de

4 Meillassoux (1992) explique en détail les mécanismes sociaux qui font que les femmes représentent, dans les sociétés traditionnelles d'Afrique de l'Ouest, "l'honneur de la famille ». Ce que Mathieu (1985 b : 16) appelle « l'arraisonnement des femmes »-1e contrôle des femmes dans leur tête et dans leur ventre, par le biais de la soumission à l'impératif de la reproduction et de la médiatisation de la conscience propre par l'écran que constitue le pouvoir physique, juridique et mental des hommes - apparaît comme une tentative de les priver de leur individualité et de leur liberté d'action et de pensée. Voilà pourquoi les femmes africaines, dont le comportement est supposé être déterminé à la fois par l'idéologie communautariste et par leur rôle sexué, partent doublement désavantagées dans un processus d'individualisation ou, pour le dire avec un terme très à la mode dans les études de psychologie et de développement, d' " empowerment ", entendant par là l'appropriation, ou la réappropriation de leur pouvoir et de leurs capacités individuelles (Rappaport, 1987 ; Zimmerman, 1990). 
façon plus ponctuelle, c'est-à-dire à la maison, lors des réunions communautaires ou, à l'occasion, sur le cours Belsunce et sur les quais du Vieux-Port. Le commerce est donc une activité « transversale », pratiquée non seulement par des commerçantes « établies », mais aussi par des femmes qui se définissent comme chômeuses ou qui ont un autre emploi, généralement dépendant. Circonscrire une «population » de migrantes sénégalaises « en commerce » m'a donc permis de confronter nombre de cas et de parcours différents dans le cadre de l'initiative économique individuelle.

J'ai rencontré sur le terrain plus d'une cinquantaine de commerçantes sénégalaises. Je suis allée vers elles, sur leurs lieux de travail, constituant ainsi un échantillon au gré des connaissances et des rencontres. J'ai interviewé de façon approfondie trente-neuf de ces femmes, récoltant autant de récits de vie de femmes qui exercent de façons diverses une activité commerciale dans la ville. La majeure partie d'entre elles (quinze) sont des commerçantes qui vendent régulièrement, même si parfois leur activité se concentre durant les mois d'été (l'hiver elles retournent au Sénégal), sur les marchés forains ; neuf d'entre elles vendent sur les trottoirs du cours Belsunce ou du Vieux-Port (deux lieux de passage intense du centre-ville) ; deux femmes ont une boutique d'artisanat sénégalais ; onze femmes pratiquent une activité commerciale chez elles ou lors des fêtes et des réunions communautaires ; enfin, deux autres femmes vendent «à la maison », mais en exerçant une activité très différente de celle des femmes que j'ai incluses dans le groupe précédent. Les premières vendent occasionnellement et de façon informelle des produits alimentaires, des habits et des bijoux qu'elles rapportent de leurs voyages au Sénégal ou au Moyen-Orient et ciblent une clientèle composée par leurs compatriotes immigrées qu'elles rencontrent lors des réunions communautaires ou qu'elles reçoivent à la maison, grâce au bouche-à-oreille. Les deuxièmes exercent des activités déclarées et stables. L'une vend de la lingerie de fabrication chinoise par Internet (à travers le site eBay) et l'autre vend des appareils ménagers à domicile, ou en organisant des réunions d'amies, d'origines diverses, à visée commerciale. Tandis que ces deux femmes, ainsi que les patronnes des boutiques d'artisanat africain ont une trentaine d'années, l'âge des autres femmes est variable : entre dix-neuf et soixante-quinze ans.

Les parcours migratoires aussi sont très différents entre eux. La majeure partie des femmes est arrivée en France dans le cadre du regroupement familial au cours des années 1980 et 1990, le plus souvent en tant qu'épouse, parfois en tant que fille d'un travailleur immigré. Aujourd'hui âgées de trente-cinq à soixante-cinq ans, ces femmes sont appelées aussi « les immigrées », en relation au statut officiel de leur mari, " travailleur immigré », qui leur donne accès aux droits sociaux et qui définit ainsi leur position sociale, leurs espaces et souvent leurs projets de vie. Bien insérées dans la communauté immigrée, elles se définissent plus comme épouses et mères de famille que comme commerçantes. Dans la pratique, elles utilisent stratégiquement la soumission à l'autorité de la famille d'origine et de leur époux comme un instrument leur permettant d'accéder à une certaine indépendance au niveau social et professionnel, sans pour autant courir le risque que leur conduite soit considérée comme " hors norme ", à savoir comme trop autonome. C'est ainsi que certaines d'entre elles ont, pour partir en France, sciemment exploité le créneau du regroupement familial. Une fois établies en France et lorsque les enfants ont grandi pour ne plus avoir besoin d'une surveillance constante, elles s'investissent souvent dans le commerce, essayant de conjuguer leur activité professionnelle et leur rôle domestique. Généralement, 
ces femmes vendent au détail sur les marchés forains et sur les trottoirs des marchandises diverses, allant de l'artisanat sénégalais à la maroquinerie de production chinoise. Elles vendent aussi souvent lors des réunions communautaires, ou encore à la maison, des tissus et des bijoux achetés en Turquie, à Dubaï ou lors d'un déplacement au Sénégal pour rendre visite à leur famille. La nature et la rentabilité de leurs activités varient selon la liberté dont elles disposent, tant sur le plan matériel, au niveau des ressources économiques et du temps libre, que sur le plan symbolique, au niveau de l'autonomie décisionnelle dont elles jouissent au sein du ménage. L'intensité avec laquelle elles s'investissent dans la profession dépend donc de leur capacité à gérer stratégiquement les contraintes domestiques et familiales.

Une autre partie importante des commerçantes que j'ai rencontrées sont venues seules en France, hors du contexte familial, au cours des années 1970 et 1980, suite à une rupture conjugale, à un veuvage ou à une situation de marginalité familiale, pour recommencer une vie tout en échappant aux contraintes sociales du pays d'origine (remariage, réinsertion dans la cellule familiale d'origine), ou simplement pour s'investir dans un projet entrepreneurial. Aujourd'hui âgées de soixante à soixante-quinze ans, elles vivent à Marseille souvent isolées, hors ménage et en marge des circuits de sociabilité de la communauté sénégalaise établie, victimes de la mauvaise réputation attribuée aux migrantes non accompagnées d'un homme en France ${ }^{5}$. Certaines ont eu un passé florissant de grossistes en objets d'artisanat africain, mais aujourd'hui la majeure partie d'entre elles vend sur les marchés forains ou dans la rue. Elles proposent sur les trottoirs de la marchandise sénégalaise (noix de cola, arachides, cure-dents, bissap ${ }^{6}$, tiouraye ${ }^{7}$, etc.) destinée à une clientèle issue de la communauté migrante. Sur les marchés forains elles sont spécialisées dans la vente de colifichets de fabrication chinoise, lunettes et sacs de contrefaçon. Généralement, leur chiffre d'affaires est très faible. Néanmoins, certaines s'impliquent aussi dans le commerce international, tandis que d'autres gèrent des activités d'importexport informel entre la France (voitures, électroménagers, fripes envoyées par container), le Sénégal (artisanat africain) et le Moyen-Orient (or et tissus). Aujourd'hui, certaines d'entre elles sont considérées, en France aussi bien qu'au pays, comme des " grandes dames » du commerce et sont prisées pour la richesse dont elles font preuve par des redistributions généreuses envers un vaste réseau de clientèle, ce qui leur permet de racheter leur position marginale.

5 Sans que cela soit forcément en relation avec leurs véritables activités, ces migrantes « seules » sont souvent accusées d'être des «femmes libres». Il s'agit d'une étiquette, dont les sens et les usages - qui font référence à une trop grande liberté des femmes par rapport au contrôle masculin, seul garant de la conformité de la conduite des femmes à la moralité communautaire mériteraient d'être discutés, mais qui est souvent utilisée comme synonyme de prostituée. Cette formule, loin de traduire une donnée objective, est l'expression de la pression communautaire sur le comportement féminin, suivant une idéologie qui présuppose que la femme doit toujours être accompagnée et contrôlée par un homme. La sexualité féminine, en d'autres termes, doit être soumise, à travers le contrôle masculin, à l'impératif de la reproduction, elle ne peut pas être libre ni s'exercer hors du cadre du mariage (Tabet, 2005). En effet, le non-respect des lois de la reproduction sociale entraînerait non seulement une perte d'effectifs pour la communauté, mais aussi une perte d'identité et de continuité socio-culturelle, d'autant plus importantes en migration que le groupe y sent son identité menacée.

6 Feuilles d'oseille rouge utilisées pour faire une boisson rafraîchissante très prisée.

7 Encens qu'elles fabriquent elles-mêmes. 
Enfin, un nombre plus restreint de femmes sont venues toutes seules pour finir leurs études et c'est parmi celles-ci qu'on trouve les femmes qui vendent occasionnellement à la maison, les patronnes de boutique et la vendeuse d'appareils électroménagers. Issues de milieux sociaux aisés, ces femmes se sont souvent mariées avec des hommes rencontrés sur place et dans la majeure partie des cas elles ont formé un ménage mixte, ayant un époux soit français, soit d'une autre origine africaine. Ainsi, ces jeunes femmes décident fréquemment de rester en France bien au-delà de la durée de leur cursus universitaire - que parfois elles ne terminent pas. À la place d'une carrière qui se situe en continuité de leur sujet d'études, elles optent pour une réorientation du parcours professionnel vers une activité entrepreneuriale et le commerce. Mais l'activité marchande qu'elles exercent est complètement différente par rapport à celle des autres commerçantes sénégalaises. En s'appuyant sur des financements obtenus de la part du conjoint (dans le cas des deux femmes interviewées, il s'agit d'un homme d'origine française), de leur famille d'origine, ou encore des circuits bancaires ou des subventions publiques, certaines de ces femmes ont monté des magasins d'art et d'artisanat africain qui ciblent une clientèle «branchée » et presque exclusivement européenne. Elles exploitent ainsi la mode de l'art africain et de l'artisanat ethnique et gèrent des chiffres d'affaires assez élevés. Elles se distinguent des autres commerçantes par l'autonomie de leurs parcours, se rapprochant de plus en plus d'un modèle " individualiste » occidental, par la typologie de leurs choix de vie, de leurs investissements entrepreneuriaux, ainsi que par la volonté de se tenir à l'écart de la vie de la communauté immigrée, qu'elles considèrent comme trop « traditionnelle ».

Du commerce à la sauvette sur les trottoirs du centre-ville, au commerce forain sur les marchés citadins, de l'import-export via container entre Afrique et Europe à la vente par Internet ou à la maison, les activités marchandes dans lesquelles les migrantes s'investissent sont aussi variées que leurs profils sociaux. Toutefois, il existe un point commun à tous ces parcours : les activités commerciales restent toujours en connexion avec la famille d'origine.

\section{DES PARCOURS DE FEMMES ENTRE ASPIRATIONS INDIVI- DUALISTES ET INSCRIPTIONS COMMUNAUTAIRES}

Pour mieux comprendre les parcours de ces migrantes, leur projet migratoire, les objectifs et les spécificités de leur investissement professionnel et pour illustrer les dynamiques qui gouvernent les relations qu'elles entretiennent avec leurs familles d'origine, je propose d'examiner l'histoire de trois d'entre elles. Celles-ci, que je présenterai sous forme de synthèse, donnent à voir de manière exemplaire la complexité, ainsi que le caractère contradictoire et contraignant des relations sociales et matérielles qui lient les migrantes à leurs familles d'origine.

Ces histoires de vie ont été récoltées dans le cadre d'interviews semi-structurées au cours desquelles j'ai demandé aux femmes de retracer leurs parcours socio-professionnels, en revenant sur les motivations qui ont inspiré la migration et sur ce qu'elles considéraient comme les éléments déterminants de leur réussite. Le récit de vie, à travers la reconstruction de la trajectoire, des événements, des états d'âme et des représentations qui l'ont balisée, s'est présenté comme le moyen le plus adapté à l'expression d'une repré- 
sentation de soi comme acteur, donnant à voir le jeu de négociation entre les aspirations à l'autonomie individuelle et la pression sociale.

Au cours de mon travail de terrain, j'ai aussi pu réaliser un suivi continu des activités professionnelles des migrantes, ainsi que de l'évolution de leur situation familiale. Des visites répétées sur les marchés, des contacts fréquents avec les femmes et leur entourage, ainsi que la participation aux réunions collectives (comme les tontines ${ }^{8}$, les fêtes religieuses et familiales), ont jalonné cette période et m'ont aussi permis d'interroger certains membres de la famille des migrantes (les époux, les enfants adolescents et adultes, les parents).

Soda a soixante-quinze ans et est arrivée en France en 1974, par bateau. Après une période de veuvage au Sénégal, elle s'est remariée avec un émigré et l'a rejoint au Havre où il était employé chez Renault. Quand ce dernier a quitté la France pour " prendre sa retraite » au Sénégal en 1981, Soda a décidé de rester en France. Le choix de vivre toute seule et loin de son époux a mis en acte une séparation voulue de sa part sans besoin de l'expliciter. Après un bref passage à Paris, Soda a déménagé à Marseille, car des voisins sénégalais lui avaient parlé de la facilité d'exercer l'activité commerciale dans cette ville, appréciée autant par son cosmopolitisme que par l'omniprésence des marchés. Déjà commerçante de tissus au Sénégal depuis l'âge de douze ans, Soda a pu mettre à profit ses compétences à Marseille, tout en bénéficiant de son insertion dans les réseaux sociaux et commerciaux mourides. Aujourd'hui elle vend sur plusieurs marchés de la ville des objets d'artisanat sénégalais qu'elle ramène de ses voyages saisonniers au pays. À côté de son travail de détaillante, Soda loue des containers qu'elle envoie au Sénégal chargés de tissus, d'appareils électroménagers, de matelas, de machines à coudre, de voitures, de casseroles, etc., une série de marchandises d'occasion vendues par la suite par sa fille qui attend les cargaisons à Dakar. Selon les rumeurs de la communauté immigrée, une grande partie des ressources que Soda investit dans son commerce viennent de son passé de prostituée, autrement dit de « femme libre ». En effet, il semble que par le passé Soda ait exercé une activité de ce type, une fois son époux parti, mais c'est justement grâce à l'éloignement du milieu familial et à la séparation avec son mari qu'elle a pu s'investir dans le commerce avec l'intensité voulue.

Soda vise à une promotion socio-économique, d'abord pour elle-même et ensuite pour toute sa famille (ses enfants et ses sœurs), qui réside au Sénégal. Issue des classes populaires et d'une famille très pauvre, elle a travaillé pour faire en sorte de ne " manquer de rien ». Elle a fait construire une maison dans un quartier populaire de Dakar où, comme elle tient à le dire, elle a fait installer l'eau courante, un téléphone et un set de meubles : elle souligne avec fierté

8 La tontine est une sorte de loterie, spécifiquement féminine, traditionnellement pratiquée dans la société sénégalaise par des femmes de tout âge et de toute origine sociale. Elle est formée d'un nombre établi de personnes qui se réunissent à une échéance fixe (la tontine à laquelle j'ai participé réunissait vingt-six femmes à une cadence mensuelle) chez l'une des membres, à tour de rôle. Après avoir partagé un moment festif, pendant lequel on mange et on danse, chaque femme verse sa cotisation (dans ce cas cent euros), qui va constituer la cagnotte. Celle-ci est gagnée par l'une des femmes présentes, dont le nom est tiré au sort. Les noms des gagnantes précédentes sont exclus du groupe, de façon à ce que chacune des femmes puisse gagner, à son tour. Une partie de la cagnotte doit être obligatoirement réinvestie dans une fête que la gagnante donnera chez elle à l'occasion de la rencontre suivante, tandis que ce qui reste peut être utilisé librement. Un grand nombre de femmes décident de l'employer comme capital de départ pour une entreprise personnelle, pour financer un voyage au Sénégal, ou encore pour payer le mariage d'une fille ou solder la dette contractée avec un fournisseur. La tontine représente une forme spécifiquement féminine de micro-crédit autofinancé, personnel et autogéré. 
qu'elle « a tout ce qu'il faut ». Le rôle de soutien qu'elle a auprès de sa famille au Sénégal est aussi d'importance capitale pour elle. Chaque mois elle envoie un mandat de 300 euros à sa fille, qui le redistribue auprès des membres de la famille élargie. Comme elle le dit « là-bas en brousse, il n'y a rien » et tout le monde compte sur son aide, même pour manger. Ainsi, la position qu'elle a acquise grâce aux redistributions de ses revenus lui a permis de se racheter d'un passé conjugal turbulent et d'une réputation douteuse, diffusée autant auprès de la communauté immigrée marseillaise que dans les cercles sociaux dont elle est originaire.

Ndèye a trente-deux ans et vit en France depuis huit ans. Elle vient de Dakar, où elle a rencontré son mari, un Français qui, à l'époque, faisait son service militaire au Sénégal et qui l'a ramenée en France pour l'épouser. Ça a été difficile de faire accepter sa décision à ses parents, car la représentation commune veut qu'une femme qui va en France pour vivre avec un mari français, qui plus est, pas de la même religion qu'elle, finira forcément dans la prostitution, pour nourrir son mari. Mais Ndèye avait déjà au Sénégal une posture indépendante et marginale au sein de sa famille car, petite fille, elle a été séparée de sa fratrie et a été confiée par ses parents à sa grand-mère, à l'époque très âgée, chose qu'elle a très mal vécue, mais qui l'a rendue rapidement autonome par rapport à ses parents. Ainsi, Ndèye ne leur a pas permis d'interférer avec sa décision d'émigrer par amour.

Après avoir travaillé pendant plusieurs années comme femme de chambre dans des hôtels marseillais, Ndèye se lance dans le commerce via Internet. Elle n'a qu'une instruction élémentaire et aucune connaissance de l'informatique quand elle commence, mais ayant entendu parler à la télévision du site eBay elle décide de se procurer un ordinateur et, en soustrayant de l'argent à son mari, achète sa première marchandise, de la lingerie chinoise. Le succès arrive subitement, les demandes d'achat se multiplient et contraignent Ndèye à agrandir sans cesse son stock. Aujourd'hui, elle vend comme grossiste sur le net; elle a un chiffre d'affaires très élevé et projette d'ouvrir une usine en Chine où elle fera produire sa propre lingerie. Pour Ndèye le travail n'est pas qu'un gagne-pain, mais représente un moyen de se sentir utile, d'avoir la sensation de faire quelque chose et de sortir de la maison.

Les retombées économiques et sociales du travail ne sont quand même pas négligeables pour elle : Ndèye a investi une bonne partie de ses revenus au Sénégal, achetant un taxiphone et montant un cybercafé où elle vend aussi des téléphones portables. Elle a impliqué son frère dans cette entreprise, en lui donnant $25 \%$ de la société. De plus, elle envoie régulièrement de l'argent à ses sœurs et à sa mère. Même si elle ne s'entend pas bien avec cette dernière, car elle ne supporte pas qu'après l'avoir abandonnée elle veuille encore lui « dicter sa vie », Ndèye se sent quand même obligée par le devoir de solidarité envers ses parents et par la volonté de maintenir sa place dans le groupe social d'origine, par le biais de la redistribution des ressources accumulées en migration. La réussite du parcours, en effet, « c'est pour le Sénégal ». L'objectif de Ndèye est de faire fructifier au maximum son entreprise de lingerie et de vendre la société quand elle aura atteint son plein épanouissement. Alors elle planifie de rentrer au Sénégal avec son mari, pour " profiter de la vie ", pour vivre en " grande dame » dans le luxe et le bien-être. Elle projette ainsi d'acheter un grand hôtel dont elle sera la gérante, tout en vivant dans la maison qu'elle aura fait construire sur le terrain qu'elle a déjà acheté près de Dakar.

Sokhna a trente ans et est venue à Marseille quand elle avait dix-sept ans, pour finir ses études supérieures. C'était une décision concertée, une volonté partagée entre elle et ses parents : elle, elle voulait « découvrir l'inconnu et la belle vie »; sa mère voulait l'envoyer en France faire fortune, mais cela Sokhna ne l'a compris qu'après coup. Une fois à Marseille, tout en étant logée chez une tante, Sokhna a beaucoup souffert du manque d'encadrement familial et de la distance avec ses parents. Elle a donc terminé le lycée, mais après deux ans d'université, elle a abandonné les études et a trouvé un petit travail comme vendeuse 
dans un magasin de sport. À l'époque, comme elle le dit non sans regret, elle avait déjà " la pression de ses parents ", surtout de sa mère, qui lui demandait constamment de lui envoyer de l'argent. Une pression qui l'a beaucoup affectée et dérangée, car elle juge injuste d'adosser la responsabilité du maintien d'une famille à une femme aussi jeune. Entre-temps Sokhna s'est mariée avec un Camerounais qu'elle a connu en France. Son père était opposé au mariage, à cause de la différence de nationalité et de religion (elle est musulmane, lui, chrétien), mais sa mère a insisté pour que le père accepte, car elle avait compris que ce mari potentiel gagnait bien sa vie.

Maintenant Sokhna travaille comme vendeuse d'appareils culinaires à domicile. Ce n'est ni le travail de ses rêves ni un travail alimentaire, parce que son mari, ingénieur électronique et propriétaire de deux salons de coiffure africaine dans le quartier de Noailles, lui offre une vie aisée. Il s'agit plutôt d'un travail qui permet à Sokhna d'avoir une activité à elle, de sortir de la maison où elle déprime, de rencontrer des gens et de se mettre à l'épreuve.

Le soutien de la famille continue de représenter un grand souci pour Sokhna. Elle envoie au Sénégal un mandat de 150 euros chaque mois, sauf dans des cas particuliers, où elle envoie davantage d'argent. Sa mère, ses frères et ses belles-sœurs ne cessent de la solliciter en lui exposant leurs problèmes économiques. Et même si cela l'agace, Sokhna n'arrive pas à « ne pas faire le geste ». Elle a l'impression qu'avec son mari ils « travaillent sans cesse et ne font que se priver ", et que de cette façon ils ne parviennent pas à avancer dans leurs projets familiaux ou à faire des économies. Pour arrêter de se faire ponctionner, Sokhna et son époux ont essayé d'investir en Afrique, d'autonomiser leurs familles en créant des opportunités de travail. Ils ont envoyé des voitures au Sénégal, pour monter une entreprise de taxis, mais le projet a été détourné et les voitures ont été prises par la famille. La même chose est arrivée avec l'argent qu'ils ont envoyé pour ouvrir un magasin d'alimentation. Ils ont cessé d'espérer que l'argent envoyé serait investi, ayant compris que les fonds alloués pour des projets entrepreneuriaux seront toujours détournés. C'est pour cela qu'aujourd'hui Sokhna s'est résignée et considère que l'argent qu'elle envoie c'est « à perdre », c'est pour la famille, qui en fera ce qu'elle veut.

Comme on le voit bien à la lecture des synthèses de ces trois récits de vie, les relations des migrantes avec leurs familles d'origine sont caractérisées par une profonde ambivalence. Afin de la comprendre et d'en saisir toutes les implications et les enjeux, il est nécessaire de s'interroger sur la force des représentations dans lesquelles elle plonge ses racines. L'intensité de ces liens s'inscrit dans le contexte social d'Afrique de l'Ouest, qui est foncièrement organisé autour de l'élément communautaire (Meillassoux, 1992 ; Marie, 1997b ; Augé, 1977). Dans le modèle idéal-typique de ces sociétés, que Dumont (1966) appelle « holistes », l'individu est « absorbé », " englobé » ${ }^{9}$ dans le « tout » qu'est le groupe social (Viti, 2005) et soumis au principe hiérarchique qui organise la solidarité communautaire. Ce principe se structure selon deux critères fondamentaux qui sont l'âge et le sexe (les aînés sont donc considérés comme supérieurs aux cadets et les hommes aux femmes (Diop, 1985). La solidarité communautaire se fonde sur le principe de la « dette infinie » (Marie, 1997a) qui lie l'individu au groupe auquel il appartient, en vertu d'un système cyclique d'avances et de redistributions (Meillassoux, 1992). L'individu est donc redevable au groupe social par le soutien matériel et l'éducation qu'il a reçus et lui est lié par une « dette » qu'il doit sans cesse rembourser (Marie, 1998) et par des rapports de dépendance personnelle. La famille étendue est le lieu où se manifeste avec le plus de force l'existence de ces rapports de dépendance (Diop, 1981 ; Viti, 2007). Les relations

9 Pour une analyse spécifique de chacun de ces termes, voir respectivement Durkheim (1991) et Dumont (1966). 
de parenté déterminent les rôles sociaux que l'individu endossera tout au long de sa vie, en définissant à la fois un champ de solidarité, de protection et d'obligations à l'intérieur duquel il sera inclus. Les individus sont ainsi socialisés dès leur naissance à un système de normes, de valeurs et de catégories de la pensée qui d'un côté les inclut dans des rapports hiérarchiques de dépendance personnelle et de l'autre véhicule un habitus anti-individualiste de solidarité et de partage communautaires, apte à intégrer et à soutenir tout membre du groupe. C'est ce que Augé (1977) a appelé le «totalitarisme lignager ». Les femmes, traditionnellement considérées comme «propriété » du groupe et dépendantes à vie, en sont les premières victimes. Le sujet communautaire donc, tout en étant individué (c'està-dire reconnu comme une personne unique, ayant des qualités et un parcours existentiel spécifiques) ne s'individualise ${ }^{10}$ et ne prend son autonomie qu'avec difficulté et au prix de lourds conflits.

Au vu de ces considérations, nombre d'auteurs se sont interrogés sur la possibilité de parler de " sujet individuel » dans les sociétés africaines contemporaines (Augé, 1977 ; Dumont, 1983 ; Marie, 1997a ; Hussein, 1993). Il faut pourtant nuancer cette lecture statique des sociétés traditionnelles d'Afrique Occidentale et prendre en compte le caractère plastique et problématique de chaque culture (Augé, 1994), ainsi qu'il est nécessaire de reconnaître qu'aujourd'hui les aspirations à l'individualisation sont bien présentes en Afrique de l'Ouest comme ailleurs, surtout en milieu urbain. Dans les sociétés postcoloniales actuelles, modernité et tradition sont en dialogue, les valeurs holistes et individualistes sont imbriquées, l'individualisation est in fieri (Veith, 2005).

L'individualisation est encouragée aussi par de nouvelles expériences existentielles. L'exode rural, la modernisation du secteur du travail, la vie urbaine, la scolarisation et les migrations, entre autres, ont conduit à l'hybridation des modes de vie ainsi que de l'organisation sociale. L'expérience de l'hétérogénéité pousse les individus à porter une réflexion critique sur leurs appartenances et leurs modes de vie, leur permettant ainsi de se constituer en tant que sujets. Cette remise en cause de l'identité personnelle, empêchant l'individu d'être totalement dans son rôle ou sa position, l'encourage à repenser et redéfinir ses solidarités communautaires. En outre, il est nécessaire de reconnaître que la profonde crise qui mine l'économie africaine porte atteinte aux circuits de redistribution communautaires, qui font forcément des exclus, induisant par contrecoup l'émergence de plus en plus diffuse de l'individualisation. Cependant, par un mouvement en sens inverse qui peut sembler paradoxal, le contexte de crise permanente et l'absence de véritables systèmes de sécurité sociale rendent davantage vital, pour l'individu, d'avoir recours aux réseaux de solidarité communautaire afin de s'en sortir au quotidien (Leimdorfer et Marie, 2003a ; Hussein, 1993), contrecarrant ainsi ses aspirations à l'autonomisation.

10 Comme je l'ai rapidement suggéré dans les pages précédentes, l'individualisation est un processus d'affranchissement qui se réalise sur deux niveaux différents. Un niveau matériel, impliquant que le sujet puisse concevoir la possibilité de survivre hors du cadre communautaire. Et un niveau intellectuel et affectif, postulant que l'individu ait la possibilité de se mettre à distance réflexive et critique des fondements éthiques qui sous-tendent les ressorts de la solidarité communautaire, pour pouvoir concevoir sa survie sociale et psychologique hors du cadre de la communauté. L'individualisation correspond alors au développement d'une conception de soi en tant qu'individu indépendant, matériellement, spirituellement et socialement, des liens, des déterminismes et des contraintes communautaires. 


\section{La migration comme ambition}

Les trajectoires biographiques des femmes dont j'ai retracé le parcours montrent que la migration est une démarche à laquelle elles participent activement, même quand elles y prennent part par le biais de moyens indirects (le mariage, les études) et sous l'impulsion de la famille. Au cœur de leurs parcours migratoires réside toujours le désir d'atteindre « quelque chose de plus ». Il s'agit de meilleures conditions de vie, qu'elles espèrent obtenir à travers l'investissement dans l'activité professionnelle, d'une promotion sociale, qui se reflète aussi bien au niveau familial qu'au niveau individuel, ou d'une reconnaissance professionnelle. C'est pour cela que les migrantes se tournent souvent vers l'activité commerciale, espérant y trouver le moyen d'une ascension sociale rapide (Peraldi, 2002b).

Bien souvent, en outre, les femmes émigrent dans une optique d'épanouissement personnel, pour s'enfuir d'une situation familiale oppressante, faisant appel à la nécessité de subvenir aux besoins de la famille, davantage pour justifier un voyage qui se fonde sur un désir individuel, que pour évoquer des nécessités les contraignant réellement à affronter un tel voyage. La migration féminine, surtout quand elle se fait « en solitaire », est difficilement acceptable dans le milieu d'origine pour les raisons que nous avons déjà évoquées. Ainsi, dans nombre de cas, ce n'est qu'après coup que les migrantes justifient leur parcours aux yeux de la famille, autrement dit ce choix solitaire est accepté seulement une fois que l'assise économique de l'émigrée s'est consolidée. Grâce à l'investissement des gains cumulés en migration dans les circuits de redistribution familiale, les migrantes arrivent soit à racheter leur position sociale d'un passé marginal, soit à acquérir un nouveau statut, moins dépendant, plus autonome et dont l'autorité est davantage respectée dans les cercles familiaux et communautaires, un statut en fin de compte plus « masculin ».

La migration, qui est souvent le fait d'individus qui sortent du cercle familial, induit en retour une accélération de l'individualisation. Cela se fait par l'éloignement des femmes du contrôle de la famille élargie, par la rencontre avec un mode d'organisation social davantage basé sur l'individualisme, mais aussi par l'investissement dans le travail indépendant. Néanmoins, comme le montrent les récits évoqués, aussi bien que nombre d'autres témoignages que j'ai pu récolter sur le terrain, pour marginale que soit la position de la femme dans son groupe social d'origine, la migration ne se fait jamais dans un vide relationnel, ni dans la rupture totale avec la famille d'origine, même si le désir d'éloignement est manifeste et, dans le même temps, entraîne un relâchement de ces liens. D'un côté, en effet, les migrantes s'appuient sur l'efficacité de réseaux migratoires rôdés, de l'autre elles conçoivent la migration toujours en relation à la famille d'origine.

\section{STRATÉGIES FAMILIALES ET INDIVIDUALISATION}

L'individualisation n'est pas seulement le fruit d'une démarche individuelle, elle semble être aussi, de surcroît, celui d'une imposition de la part du groupe familial d'origine, qui néanmoins en entrave l'aboutissement complet. Nous nous trouvons face à des injonctions contradictoires, un double-bind qui pose les migrantes dans une position complexe. Cela est évident quand on examine de près leurs parcours. 
Le témoignage de Sokhna, par exemple, souligne les contradictions générées au quotidien par le principe de la solidarité communautaire. Après avoir été envoyée en France, où elle a beaucoup souffert de la solitude et de l'éloignement de sa famille, Sokhna se trouve très tôt confrontée aux demandes pressantes d'aide économique de sa mère. Se sentant vite oppressée, elle essaye de transformer une partie de ces transferts d'argent en des projets de partenariats entrepreneuriaux avec la famille restée au pays, visant autant à améliorer ses conditions économiques et à l'autonomiser par rapport à la migrante, qu'à offrir un retour économique à cette dernière par rapport à toutes les ressources qu'elle transfère dans ces versements. Mais ces projets échouent de façon tellement décevante qu'elle finit par affirmer que l'argent envoyé au Sénégal est du capital jeté, car au final il est invariablement absorbé par les circuits de la redistribution communautaire. Sokhna remarque en effet que ces fonds ne servent pas seulement à maintenir une famille en difficulté, mais à lui garantir des dépenses ostentatoires et une place sociale élevée, échouant dans leur fonction d'autonomisation. Tout en étant consciente et indignée du mécanisme d'exploitation auquel ses revenus sont exposés, Sokhna n'est pas capable de s'en soustraire. Elle n'arrive pas à ne pas obéir aux injonctions de la solidarité communautaire qu'elle a si profondément incorporées.

Par le biais de ses requêtes continuelles, la famille d'origine semble presser d'absorber la « réussite » de l'individu, sans lui permettre de continuer son parcours indépendant, le même qu'elle a impulsé, peut-être involontairement, envoyant la femme en migration. La totale indépendance de l'individu est ainsi niée par le groupe d'origine qui demande et présuppose pour soi une part des richesses du migrant, refusant bien souvent de les investir en une entreprise génératrice de revenus et potentiellement porteuse d'autonomie. Reposant sur la contrainte de réponse des migrantes aux obligations communautaires, une culture de l'assistanat se développe, qui parfois légitime le fait que les individus restés au pays n'investissent pas dans des activités professionnelles. Il s'agit d'une logique qui va bien au-delà d'une forme d'aide et qui s'inscrit dans une dynamique d'échange jamais quantifiable et par conséquent déséquilibré. Devant être continuellement renouvelée, la « dette infinie » se transforme en un devoir de contribution illimité qui va au-delà des nécessités de la subsistance pour investir le champ des biens ou des « besoins de luxe » (les voitures, les téléphones portables, le paiement de fêtes de plus en plus fastueuses, etc.) dont l'étendue potentielle n'a pas de limite. C'est là l'un des effets les plus pervers de la captation de la migration dans la logique communautaire. Les familles envoient les individus loin du groupe pour se qualifier et/ou pour travailler, leur imposant au passage d'accumuler des ressources en se rendant indépendants, car ils se retrouvent seuls, loin de leur milieu d'origine, ne pouvant souvent pas compter sur le soutien de la famille élargie dans le pays d'immigration (soutien qui ne peut pas être remplacé par celui de la communauté immigrée, faute de moyens et de liens assez étroits), pour engloutir ensuite les fruits de leur travail et de leur individualisation, les empêchant ainsi de porter jusqu'à son terme leur démarche d'autonomisation et les insérant dans un rapport de dépendance qui est également contraignant pour les deux parties qui y participent.

Pour revenir au cas cité, Sokhna, comme bon nombre d'autres migrantes, travaille donc en partie pour sa famille d'origine et en partie pour soi et pour la famille qu'elle a fondée. Sa démarche d'individualisation la pousse à remettre en question le fonctionnement de la logique communautaire, mais elle n'est pas assez indépendante par rapport 
à son groupe d'origine et à sa socialisation pour pouvoir s'affranchir de l'obligation de donner sans compter.

Cette dynamique a été relevée aussi dans d'autres travaux. Quiminal (1998), dans une étude sur les rapports entre les migrants maliens et leurs villages d'origine, note que les migrants restent, de plus en plus logtemps, en France, commencent à porter un regard critique sur l'économie de subsistance - qu'ils contribuent à maintenir par leurs remises - et sur son mécanisme de reproduction, de plus en plus tributaire des migrants, en vertu de leur devoir de remboursement de la « dette » qu'ils ont envers leurs parents. Les demandes d'envoi d'argent adressées par les patriarches à leurs migrants sont de plus en plus importantes. Pour les migrants, l'utilisation de cet argent est incontrôlable et souvent, peu légitime, étant fréquemment employé pour des dépenses somptuaires, pour le prix de la dot pour une nouvelle épouse (Quiminal, 2009). Dans le cas étudié par Quiminal, à la différence de ce que j'ai pu observer à Marseille, les ressortissants d'un village déterminé mettent en œuvre des logiques de résistance collective, se réunissant en associations qui se proposent de transformer le village et son économie afin que les migrants ne soient plus les bailleurs de fonds des agriculteurs. De plus, le village se substitue en tant qu'interlocuteur aux familles ; un détour qui permet aux migrants de s'assurer que l'argent qu'ils envoient n'est pas utilisé pour des dépenses ostentatoires, mais sert à transformer le système de production économique et à réaliser des projets de développement à l'avantage de la communauté. Pour ce que j'ai pu observer, les migrantes sénégalaises à Marseille ne s'associent pas, chacune essayant de subvertir l'ordre déterminé de façon subjective en négociant avec la famille d'origine, au coup par coup, entreprise qui se révèle bien plus difficile.

Je propose d'élargir les propos de Quiminal au-delà du contexte de l'économie de subsistance pour voir dans ce mécanisme de ponction des migrants une dynamique diffuse, non dépendante de l'économie dans laquelle elle s'inscrit, puisqu'on la retrouve aussi en ville où les familles vivent dans une économie de marché. Je fais l'hypothèse que cette dynamique est inhérente aux rapports entre les migrants d'Afrique de l'Ouest et leurs milieux d'origine, dans la mesure où ceux-ci se trouvent, presque toujours, dans une situation de pauvreté et de manque de ressources. Qui plus est, elle se greffe sur une diffusion capillaire des rapports clientélistes et de dépendance personnelle qui perdurent, aussi, à cause du manque d'accès aux droits sociaux et de la difficulté d'insertion dans le système salarial qui pourrait les garantir en associant automatiquement le travail aux droits.

Gubert (2008) note aussi que les envois d'argent des migrants maliens ont une «fonction-assurance » pour les familles et pour les villages entiers qui les reçoivent. De cette façon, la migration assure la sécurité à long terme de la famille, bien plus que l'économie domestique, à laquelle elle se substitue dans cette fonction. Gubert remarque aussi que les remises de la migration, jouant comme assurance, induisent une baisse de la production et de l'efficacité productive des ménages qui les reçoivent : l'argent des mandats n'est pas utilisé pour effectuer des investissements productifs ou comme capital. 


\section{DES TENTATIVES DE RÉINTERPRÉTER LES OBLIGATIONS DE SOLIDARITÉ}

Se plaçant du point de vue des migrantes on remarque la tension qui naît de la tentative de faire coïncider, par divers projets souvent élaborés en collaboration avec leur famille d'origine, leurs multiples rôles sociaux. Celui de sœur ou fille émigrée, devant assistance et soutien à sa famille élargie ; celui d'épouse et mère dans le cadre d'une famille qui vit et évolue en France ; celui d'entrepreneure moderne, impliquée dans des activités économiques à visée capitaliste. Dans le choc entre les différents systèmes de représentation auxquels ces rôles font référence, nombre de tentatives échouent, les familles d'origine finissant par absorber les ressources investies par l'individu dans les rouages des dépenses ostentatoires et de la redistribution communautaire.

La logique du travail-contrat qui affranchit, source de droits et d'autonomie et celle du travail comme capitalisation individualiste se heurtent en effet à celle du travail comme ressource communautaire, comme service qui s'inscrit dans des formes de dépendance personnelle. Cette collision produit des échecs d'autant plus pénibles pour les migrantes qu'elles y investissent à chaque fois les fruits de leur travail en France et les espoirs de voir une famille d'origine finalement indépendante et non plus pesant sur le migrant qui s'en éloigne.

Ainsi, les tentatives des migrantes de réinterpréter leurs solidarités originaires comme des investissements et non pas comme des simples " devoirs » à fonds perdu, se heurtent à l'échec de l'autonomisation du secteur économique africain. Ces tentatives de réinvestir les revenus, de soutenir la famille et les «clients » tout en les autonomisant et, pourquoi pas, en produisant des bénéfices ultérieurs, manifestent la volonté des migrantes d'étendre l'esprit d'individualisation et d'entreprise à leur contexte d'origine, afin de subvertir les logiques de redistribution qui les dominent et de gagner un plus grand espace d'autonomie pour soi et pour la famille qu'elles ont fondée.

Comme le note Streiff-Fenart (2006), la migration induit un parcours d'individualisation qui amène le migrant à reporter sur la promotion sociale de ses enfants les investissements qu'avant il consacrait à la subsistance de la famille étendue restée au pays. Ses dépenses se réorientent ainsi vers le pays d'immigration et en faveur de son ménage, tandis que sur le plan culturel on observe une prise de distance vis-à-vis des hiérarchies sociales traditionnelles. Les témoignages rapportés illustrent que ce processus s'accompagne de négociations continuelles engendrant souvent des conflits et des tensions, des tentatives d'ajustement concernant le montant à envoyer, s'appuyant parfois sur une dissimulation de la condition réelle de vie de la migrante en France, ainsi que des tentatives de réduire le nombre des destinataires (les migrantes essayent de quantifier les remises pour qu'elles ne répondent qu'aux besoins de la famille nucléaire dont elles sont issues) et la fréquence des envois. Certains de ces conflits débouchent sur de véritables disputes qui opposent la migrante à sa famille d'origine, d'autres engendrent des tensions qui restent en sourdine et qui rendent tendus les rapports à distance.

Mais les conflits se répercutent aussi au niveau des ménages en France, lorsque les migrantes vivent en couple ou en famille. En effet, souvent les époux ont des projets, 
comme l'achat d'une voiture, d'une maison, des dépenses liées aux études des enfants, dans le cadre desquels la contribution économique de la femme a un rôle important. Traditionnellement, les revenus de la femme ne reviennent qu'à elle, qui peut en disposer comme bon lui semble, pour ses besoins ou pour ceux de ses enfants. Avec le changement des rôles induit par la migration et l'investissement professionnel des femmes, celles-ci jouissent non seulement d'une certaine autonomie financière, mais aussi d'un rôle plus actif dans l'économie du ménage. Dans les foyers où les deux époux sont sénégalais, ou africains, chacun des deux envoie une partie des ressources du ménage à sa famille d'origine, les soustrayant ainsi au développement de ce dernier. La pression financière est objet de tensions, mais elle est partagée et les époux en général s'y opposent et essayent de la limiter ensemble. Dans les foyers où l'un des époux est français, au contraire, la pression financière de la part de la famille d'origine est rarement comprise et acceptée et elle devient une source de conflit entre les conjoints.

Toutefois, si les migrantes continuent d'envoyer une partie importante de leurs revenus à la famille, ce n'est pas seulement parce que ceux-ci constituent la représentation matérielle de leur insertion dans le groupe social, les gages de leur identification sociale s'appuyant sur leur socialisation. C'est aussi parce que cela représente une autre forme de capitalisation, un investissement qui n'a pas un visage immédiatement économique, mais qui équivaut à une forme d'assurance sociale. Aucune femme rencontrée, même marginale dans son milieu d'origine au moment du départ, ne s'est séparée définitivement de sa famille d'origine, en rompant tout lien préexistant à la migration. Au contraire, comme je l'ai déjà mentionné, le « partage » de revenus de la migration a été le moyen de se réinsérer dans le groupe et d'y consolider sa place.

Les migrantes investissent dans les cercles communautaires sénégalais aussi parce que maintenir un vaste réseau de clients signifie entretenir un réseau de débiteurs potentiels chez lesquels elles pourront chercher du soutien en cas de difficulté. Il s'agit donc d'une capitalisation symbolique et sociale qui a aussi une fonction économique latente. Les migrantes s'inscrivent ainsi activement dans un double système social, le système « moderne », a-personnel, basé sur les droits individuels acquis par le travail, et le système " communautaire ", basé sur des rapports de dépendance personnels et sur les statuts attribués par naissance. Elles recherchent aussi une double promotion sociale : une promotion personnelle, inscrite dans une logique individualiste et capitaliste et une promotion sociale " communautaire », inscrite dans une logique clientéliste et de redistribution.

\section{DIFFÉRENTS SENS ET ISSUES DES ACTIVITÉS COMMER- CIALES}

Le projet professionnel constitue souvent le pivot de la migration. Dans nombre de cas, il fait partie de son dessein initial. Car le travail représente le moyen fondamental pour réaliser cette ascension sociale dont la migration est considérée comme porteuse. Même quand le travail indépendant ne fait pas l'objet d'une formulation et d'une organisation préalables à la migration, il représente une éventualité qui est toujours prise en compte, voire un aboutissement que les femmes se proposent d'atteindre. Le commerce, 
pour les migrantes sénégalaises, ne représente pas, ou pas seulement, un repli dicté par la nécessité, par le manque de qualifications ou, au contraire, face à la discrimination qu'elles rencontrent à l'insertion dans le monde du travail français qui ne valorise pas comme il le faudrait leurs compétences. L'investissement dans l'activité marchande est bien souvent un choix, un désir. En effet, en tant que travail autonome, il donne droit à une triple forme de reconnaissance. Une reconnaissance économique, si l'activité marche bien et qu'elle rapporte des revenus. Une reconnaissance sociale, permettant à la migrante d'entrer dans des cercles de sociabilité du contexte local (comme ceux des colporteurs, des forains, etc.), mais aussi de consolider une position sociale reconnue et appréciée en tant que commerçante, voire « grande » commerçante dans la communauté migrante. Enfin une reconnaissance individuelle, offrant à la femme un champ d'action où elle peut faire la preuve de ses capacités. Il n'est donc pas exclusivement le fait de femmes en situation de nécessité économique, mais un créneau dans lequel s'investissent des femmes qui, comme Ndèye par exemple, cherchent un moyen de réalisation personnelle et de reconnaissance individuelle.

L'investissement des migrantes dans l'activité marchande se situe souvent en continuité avec des activités qu'elles ont exercées dans leur pays d'origine. Son déroulement profite ainsi de la mise en valeur de savoir-faire et de compétences déjà développés et testés sur le terrain. Même quand il n'est pas le prolongement d'une activité déjà menée, l'exercice du commerce se situe en continuité avec la tradition marchande féminine qui est très diffusée en Afrique de l'Ouest (Lambert, 1993 ; Cordonnier, 1987 ; Clark, 1994 ; Bouly de Lesdain, 1999 ; Lecarme-Frassy, 2000). Cependant, en interrogeant plus en profondeur cette continuité, nous pouvons observer que l'activité marchande des migrantes a des caractéristiques spécifiques qui la différencient du commerce féminin en Afrique. En effet, les capacités d'accumulation de la petite entreprise commerciale africaine ont été décrites comme profondément biaisées par la logique contraignante des redistributions communautaires et, de ce fait, nombre de ces petites entreprises ne peuvent pas être considérées comme véritablement capitalistes (MacGaffey, 1988 ; Grégoire et Labazée, 1993 ; Sarr, 1998 ; Lecarme-Frassy, 2000 ; Lautier, 2004). L’analyse des parcours des migrantes sénégalaises à Marseille nous montre au contraire que leurs activités sont souvent organisées dans un esprit capitaliste. Si nous considérons la définition « minimale » que Max Weber donne de l'ethos capitaliste, à savoir l'investissement dans une entreprise rationnelle, pacifique et continue, visant un profit toujours renouvelé (Weber, 2004), le comportement économique des migrantes sénégalaises à Marseille semble tout à fait y correspondre.

On a souvent parlé, à propos des migrants impliqués dans des activités commerciales de ce type, d' « entrepreneurs sans entreprise » (Granovetter, 1995). Cette expression a été introduite par Clifford Geertz dans une étude consacrée à l'activité des « colporteurs musulmans » en Indonésie (Geertz, 1963). Dans ce contexte, l'auteur remarque que ce qui manque à ces businessmen ne sont ni les moyens, ni l'ethos capitaliste dans sa version weberienne, mais la capacité de former des institutions économiques efficientes, des entreprises, des firmes. C'est le rapport avec les obligations de redistribution communautaire, à savoir la spécificité des liens sociaux que ces commerçants entretiennent avec leurs familles et leurs communautés d'origine, qui empêche leurs entreprises de sortir d'une économie de « marchés ». 
Nous pouvons observer la même chose pour les commerçantes sénégalaises de Marseille. Comme nous l'avons dit, leurs activités économiques font référence à deux logiques contradictoires. D'un côté elles s'inscrivent dans une optique individualiste et capitaliste, en ce qu'elles visent le profit et l'accumulation, de manière constante et à l'avantage de l'entrepreneur. D'un autre côté, la capitalisation reste liée et limitée par les obligations de la redistribution communautaire. L'antinomie entre ces deux logiques se situe au cœur des activités commerciales des migrantes sénégalaises et en détermine le déroulement et les issues qui sont différentes et dépendent des arrangements et des formes de négociations mises en œuvre par les commerçantes.

Le développement de véritables entreprises dépend en effet des fonds investis, c'est-à-dire de la capacité d'accumulation des migrantes, qui n'est possible que grâce à la soustraction d'une partie importante de leurs revenus aux circuits de la redistribution communautaire. Cela implique une réorganisation de l'étendue des obligations de la solidarité familiale, nécessaire afin que la réussite de l'entreprise individuelle de la migrante ne soit pas neutralisée par le groupe d'origine.

Ainsi par exemple, Ndèye, qui se situe dans une position de relative indépendance par rapport à son cercle familial, réussit à porter des projets entrepreneuriaux qui visent la constitution d'une véritable entreprise, une « firme ». Soda aussi réussit à développer ses affaires comme elle le veut, mais pour des raisons différentes. En effet, elle peut se permettre d'allouer toutes ses ressources d'une part à la réalisation de projets au Sénégal et de l'autre à la redistribution communautaire, car elle met complètement entre parenthèses sa vie à Marseille. Sans famille ni investissement d'aucune sorte en France, vivant dans des logements précaires et dans des conditions d'indigence à Marseille, elle destine tous ses revenus au Sénégal. Cependant, elle a su gérer l'étendue des redistributions obligatoires de façon à se laisser la possibilité d'accumuler assez d'économies pour prolonger son commerce en une activité d'import-export à laquelle participe sa famille d'un côté, et pour démarrer une entreprise de taxi qu'elle a confiée à son mari et à son fils de l'autre. Sokhna, au contraire, désire garder une partie de ses économies pour ses projets de vie en France, c'est-à-dire pour soi et pour sa famille nucléaire et cherche, à travers des projets d'autonomisation de la famille d'origine, une issue qui puisse convenir aux deux parties. Pourtant ses tentatives de créer des partenariats impliquant des membres de sa famille au Sénégal ont, jusqu'à aujourd'hui, échoué à cause de l'inertie des logiques communautaires de gestion des ressources. Se retrouvant toujours contrainte de répondre aux demandes pressantes de sa famille d'origine, elle n'arrive pas à développer ses projets personnels et familiaux. 


\section{CONCLUSION}

Dans la migration se confrontent deux modalités différentes pour interpréter le lien social et l'action économique, et avec ceux-ci la migration même et ses issues : une modalité individualiste et une modalité communautaire. La superposition de ces deux logiques génère des tensions qui affectent profondément les trajectoires des migrantes.

Bien qu'impliquées dans des parcours solitaires et/ou individualistes et aspirant à une réussite personnelle, les migrantes sénégalaises consacrent une partie importante de leurs revenus aux remises en faveur de la famille d'origine qui est restée au pays. Elles envoient des sommes d'argent tous les mois, elles achètent et expédient les biens qu'on leur demande (des habits, des appareils électroménagers, des voitures), elles offrent de l'argent lors des visites rituelles quand elles rentrent au Sénégal, des visites où les sollicitations des parents, des amis et des voisins sont aussi escomptées que non refusables.

La rhétorique de l'aide familiale est un leitmotiv dans les discours des migrantes : c'est ce qui justifie le déplacement et l'éloignement du cadre familial, mais aussi ce qui mesure le succès du parcours migratoire et entrepreneurial. Ainsi, pour les migrantes comme pour leurs familles, celui-ci dépend à la fois des acquis personnels et de la capacité de redistribution dont les femmes font preuve. La réussite est la reconnaissance de la « puissance » de la migrante, dans laquelle le facteur économique et le facteur social sont profondément liés. En effet, le succès économique n'est socialement reconnu que s'il est employé, au moins en partie, dans des redistributions en faveur du groupe d'origine. Car l'assise économique, en ce qu'elle implique la possibilité et l'obligation de redistribuer, est synonyme de puissance sociale, une puissance qui se manifeste dans la possibilité et dans la capacité de gérer un vaste réseau de clientèle. Ce sont donc à la fois la migrante, la famille qu'elle a fondée en France et sa famille d'origine qui bénéficient des changements et des opportunités offertes par la migration, bien que de façons différentes.

En conclusion, nous pouvons affirmer que la trajectoire migratoire et professionnelle des migrantes se fait en articulant des aspirations à l'autonomie et à l'indépendance avec une adhésion retravaillée à la logique de la solidarité communautaire. Au centre de ces logiques contradictoires, au cœur de cette ambivalence que la migration encourage et accentue, les femmes émergent comme sujets autonomes, réorganisant et articulant les sphères de valeurs dans lesquelles elles s'inscrivent et les obligations de solidarité liées à leur rôle social (Kaufmann, 2001 ; Lahire, 2001 ; Dubar, 2005). Comme l'ont remarqué d'autres auteurs aussi, l'individualisation des femmes, et en particulier des migrantes, « est un processus non linéaire, fragile et inachevé » (Veith, $2005: 61$ ). Les tensions entre références communautaires et références individualistes, déjà expérimentées dans le pays d'origine, mais amplifiées dans le contexte migratoire, ouvrent des brèches qui offrent aux migrantes un espace pour changer leur statut, essayer de renégocier les rapports sociaux dans lesquels elles s'insèrent et, à terme, par les ajustements et les remaniements qu'elles mettent en œuvre, devenir porteuses de changement, pour la société et pour l'individu (Cuche, 2009). 


\section{Références bibliographiques}

AUGÉ Marc (1994) Le sens des autres. Actualité de l'anthropologie, Paris, Fayard, 199 p.

AUGÉ Marc (1977) Pouvoirs de vie, pouvoirs de mort, Paris, Flammarion, 216 p.

AFRICAN DEVELOPPEMENT BANK (AFDB) (2007) Migrant Remittances a Development Challenge, [en ligne]. URL : http:/www.afdb.org/fileadmin/uploads/afdb/Documents/ Publications/70000012_EN_Transfert\%20des\%20fonds\%20ANG.pdf

BAVA Sophie (2003) De la « baraka » aux affaires : ethos économico-religieux et transnationalité chez les migrants sénégalais mourides, Revue Européenne des Migrations Internationales, 19 (2), pp. 69-84.

BERTONCELLO Brigitte et BREDELOUP Sylvie (1999) Le Marseille des marins africains, Revue Européenne des Migrations Internationales, 15 (3), pp. 177-197.

BLANCHARD Melissa (2008) Migrantes sénégalaises « en solitaire » à Marseille. Des statuts ambigus entre marginalité et quête de reconnaissance, Diasporas, Histoire et sociétés, 11, pp. 111-122.

BLANCHARD Pascal et BOËTSCH Gilles (2005) Marseille Porte Sud, Marseille-Paris, La Découverte, $239 \mathrm{p}$.

BOULY DE LESDAIN Sophie (1999) Femmes camerounaises en région parisienne : trajectoires migratoires et réseaux d'approvisionnement, Paris, L'Harmattan, 242 p.

BREDELOUP Sylvie (2001) Marseille, carrefour des migrations africaines, in Michel Peraldi Éd., Cabas et containers, Paris, Maissonneuve et Larose, pp. 65-106.

CLARK Gracia (1994) Onions are my husband. Survival and Accumulation by West African Market Women, Chicago, The University of Chicago Press, 464 p.

CORDONNIER Rita (1987) Femmes africaines et commerce. Les revendeuses de tissu de la ville de Lomé (Togo), Paris, L'Harmattan, $190 \mathrm{p}$.

DAUM Christophe (1998) Les associations de Maliens en France : migrations, développement et citoyenneté, Paris, Karthala, 253 p.

DAUM Christophe, DIARRA Hamedy et GONIN Patrick (1988) La fonction émigrée dans les stratégies de développement, Fontenay-aux-Roses/Paris, ENS, $117 \mathrm{p}$.

DIOP Abdoulaye-Bara (1985) La famille wolof, Paris, Karthala, 272 p.

DIOP Abdoulaye-Bara (1981) La société wolof. Les systèmes d'inégalité et de domination, Paris, Karthala, 355 p.

DIOP Momar-Coumba (Éd.) (2008) Le Sénégal des migrations, Paris, Crepos/Karthala/ONU Habitat, 434 p.

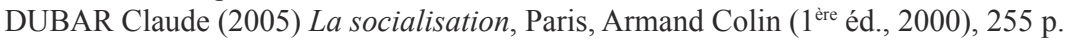

DUMONT Louis (1983) Essais sur l'individualisme. Une perspective anthropologique sur l'idéologie moderne, Paris, Seuil, 267 p.

DUMONT Louis (1966) Homo hierarchicus, Paris, Gallimard, 445 p.

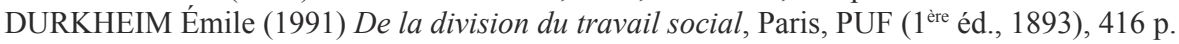

FALL Abdou Salam (1993) Relations à distance des migrants et réseaux d'insertion à Dakar, Bulletin de l'APAD, 5, [en ligne]. URL : http://apad.revues.org/document3213.html

GEERTZ Clifford (1963) Peddlers and Princes: Social Development and Economic Change in Two Indonesian Towns, Chicago, University of Chicago Press, 162 p.

GONIN Patrick (2005) Jeux d'acteurs et enjeux territoriaux : quelles migrations pour quel développement? L'exemple du bassin du fleuve Sénégal (République du Mali), in Mohamed Charef et Patrick Gonin Éds., Emigrés-Immigrés dans le développement local, Agadir, ORMES/Sud Contact, pp. 259-278.

GONIN Patrick (2001) Migrations développement : les utopies des années quatre-vingt-dix, in Reynald Blion et Isabelle Rigoni Éds., D'un voyage à l'autre. Des voix de l'immigration pour un développement pluriel, Paris, Karthala, pp. 25-48. 
GONIN Patrick et LACROIX Thomas (2005) Migrations et développement : faux semblants et vrais enjeux, Géographes Associés, 29, pp. 93-104.

GRANOVETTER Mark (1995) The Economic Sociology of Firms and Entrepreneurs, in Alejandro Portes Ed., The Economic Sociology of Immigration, New York, Russel Sage Foundation, pp. 128-165.

GRÉGOIRE Emmanuel et LABAZÉE Pascal (Éds.) (1993) Grands commerçants d'Afrique de l'Ouest : Logiques et pratiques d'un groupe d'hommes d'affaires contemporains, Paris, Karthala-ORSTOM, 264 p.

GUBERT Flore (2008) (In)cohérence des politiques migratoires et de codéveloppement françaises. Illustrations maliennes, Politique africaine, 109, pp. 42-55.

HUSSEIN Mahmoud (1993) Versant sud de la liberté, Paris, La Découverte (1 ${ }^{\text {ère }}$ éd., 1989), 190 p.

KAUFMANN Jean-Claude (2001) Ego. Pour une sociologie de l'individu, Paris, Nathan, 288 p.

LA BROSSE Renaud et MBAYE Babacar (Éds.) (1994), Migration et coopération internationale : quand les Sahéliens de l'extérieur font vivre leurs villages d'origine, Paris, Institut Panos, $42 \mathrm{p}$.

LAHIRE Bernand (2001) L'homme pluriel, Paris, Nathan, 392 p.

LAMBERT Agnès (1993) Les commerçantes maliennes du chemin de fer Dakar-Bamako, in Emmanuel Grégoire et Pascal Labazée Éds, Grands commerçants d'Afrique de l'Ouest, Paris, Karthala-Orstom, pp. 37-70.

LAUTIER Bruno (2004) L'économie informelle dans le tiers monde, Paris, La Découverte, $121 \mathrm{p}$.

LECARME-FRASSY Mirelle (2000) Marchandes dakaroises entre maison et marché, Paris, L'Harmattan, $267 \mathrm{p}$.

MACGAFFEY Janet (1988) Entrepreneurs and Parasites: The Struggle for Indigenous Capitalism in Zaïre, Cambridge, Cambridge University Press, $241 \mathrm{p}$.

MARIE Alain (1998) L'échange : sous le don, la dette, Sciences Humaines, 23 (Anthropologie. Nouveaux terrains. Nouveaux objets), pp. 28-31.

MARIE Alain (Éd.) (1997a) L'Afrique des individus. Itinéraires citadins dans l'Afrique contemporaine, Paris, Karthala, 438 p.

MARIE Alain (1997b) Du sujet communautaire au sujet individuel. Une lecture anthropologique de la réalité africaine contemporaine, in Alain Marie Éd., L'Afrique des individus, Paris, Karthala, pp. 53-110.

MEILLASSOUX Claude (1992) Femmes, greniers et capitaux, Paris, L'Harmattan (1 ${ }^{\text {ère }}$ éd., 1975), $251 \mathrm{p}$.

PERALDI Michel (Éd.) (2002a) La fin des norias ? Réseaux migrants dans les économies marchandes en Méditerranée, Paris, Maisonneuve et Larose, 495 p.

PERALDI Michel (2002 b) Introduction, in Michel Peraldi Éd., La fin des norias ? Réseaux migrants dans les économies marchandes en Méditerranée, Paris, Maisonneuve et Larose, pp. 11-42.

PERALDI Michel (Éd.) (2001) Cabas et containers: Activités marchandes informelles et réseaux migrants transfrontaliers, Paris, Maisonneuve et Larose, $361 \mathrm{p}$.

QUIMINAL Catherine (2009) Entreprendre une anthropologie des migrations : retour sur un terrain, Revue Européenne des Migrations Internationales, 25 (3), pp. 115-132.

QUIMINAL Catherine (1998) Familles, projets et capitaux, in Bernard Schlemmer Éd., Terrains et engagements de Claude Meillassoux, Paris, Karthala, pp. 285-292.

QUIMINAL Catherine (1991) Gens d'ici, gens d'ailleurs, Paris, C. Bourgeois, 222 p.

RAPPAPORT Julian (1987) Terms of empowerment/exemplars of prevention: toward a theory for community psychology, American Journal of Community Psychology, 15, pp. 121-148.

SARR Fatou (1998) L'entrepreneuriat féminin au Sénégal, Paris, L'Harmattan, 301 p.

SCHMIDZ Jean (2008) Migrants ouest-africains vers l'Europe : historicité et espaces moraux, Politique africaine, 109, pp. 5-15.

TABET Paola (2005) La grande arnaque: Sexualité des femmes et échange économico-sexuel, Paris, L'Harmattan, 207 p. 
VEITH Blandine (2005) Engagement associatif et individuation des femmes migrantes, Revue Européenne des Migrations Internationales, 21 (3), pp. 53-67.

VITI Fabio (2005) Cosa resta della schiavitù? Continuità e rotture nei rapporti di dipendenza personale in Africa Occidentale, in Pier Giorgio Solinas Ed., La dipendenza. Antropologia delle relazioni di dominio, Lecce, Argo, pp. 69-98.

VITI Fabio (2007) Schiavi, servi e dipendenti, Milano, Raffaello Cortina Editore, 298 p.

WEBER Max (2004) L'éthique protestante et l'esprit du capitalisme, Paris, Gallimard (1 ère éd. 1905), $531 \mathrm{p}$.

ZIMMERMAN Marc A. (1990) Taking aim on empowerment research: On the distinction between individual and psychological concepts, American Journal of Psychology, 18, pp. 169-177. 